\title{
arthritis + rheuma
}

\section{Zeitschrift für Orthopädie und Rheumatologie}

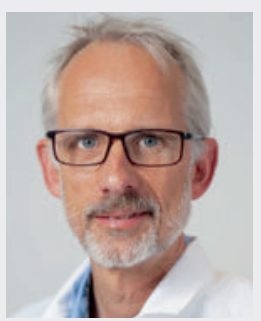

Prof. Dr. Christoph Fiehn

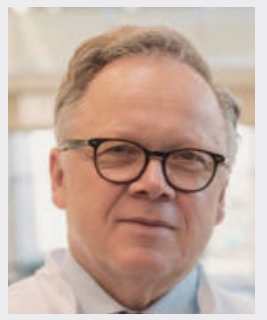

Prof. Dr. Heinz-Jürgen Lakomek

\section{Update Leitlinien 2020 - was Rheumatologen betrifft}

Leitlinien sind Empfehlungen für medizinische Standardsituationen, welche nach den Regeln der Evidenz-basierten Medizin entwickelt wurden. Sie und die darin enthaltenen Algorithmen dienen der Standardisierung von Abläufen und sind daher eine wesentliche Säule der Qualitätssicherung in der Medizin. Im Gegensatz zu reinen Empfehlungen müssen Leitlinien auf dem systematischen Studium der wissenschaftlichen Evidenz und dem Konsens von Experten beruhen. Ihre Erarbeitung folgt strengen Qualitätsauflagen, welche gewährleisten, dass entweder nur gesichertes Wissen in die Empfehlungen eingeht, oder aber Empfehlungen mit geringerem Evidenzgrad klar als diese gekennzeichnet sind. Einflüsse durch Interessenkonflikte und nicht belegtes tradiertes Wissen durch „Schulen“ oder „Eminenzen“ werden so weitestgehend ausgeschaltet. Außerdem werden Leitlinien regelmäßig überarbeitet und aktualisiert. Aus diesen Gründen spielen Leitlinien in der Medizin heutzutage eine bedeutende Rolle. Leitlinien garantieren nicht nur eine Behandlung auf einem verlässlich hohen Qualitätsstandard, sondern dienen auch der Wirtschaftlichkeit in Zeiten einer gewachsenen Auswahl von verschiedenen Behandlungsmöglichkeiten für den klinisch tätigen Arzt. In den Tabellen 1 und 2 auf der übernächsten Seite ist ein Überblick über die Entwicklungsstufen der Leitlinien sowie die jeweiligen Evidenz- und Empfehlungsgrade der in den Leitlinien enthaltenen Empfehlungen zusammenfassend dargestellt.

Leitlinien sind aber keinesfalls stets streng einzuhaltende Regeln, sondern sie dienen zur Orientierung und bilden vor allem die häufigsten Situationen (Handlungskorridor) ab. Der individuelle Patient und die individuelle klinische Situation lässt immer die Abweichung vom Algorithmus der Leitlinie zu. In solchen Fällen ist dann aber - auch aus juristischen Gründen - die Dokumentation besonders wichtig. Im Fall eines Konfliktes, z. B. mit einem Kostenträger oder einem Patienten, muss die Abweichung plausibel begründbar sein.

Wir wollen diese Ausgabe der arthritis + rheuma neuen Leitlinien mit Bedeutung für die Praxis des internistischen und orthopädischen Rheumatologen widmen. Dabei wollen wir auch über den Tellerrand blicken. Die neue S2e-Leitlinie von 2018 zur Behandlung der rheumatoiden Arthritis mit DMARD wird mit Fokus auf die neuesten Aspekte vom Autor zusammengefasst. Zur Osteoporose, auch ein stets gegenwärtiges klinisches Thema, ist ebenfalls 2017 Neues erschienen. Verena Buschhorn-Milberger et al. aus Herne und Frankfurt berichten über das Wichtigste daraus. Eng verwandt, gewissermaßen die Kehrseite der Therapie der Osteoporose, ist das Thema der Medikamenten-induzierten Kiefernekrosen. Sebastian Hoefert aus Tübingen führt uns darin ein und gibt Einblicke in ein anderes Fachgebiet, welches unsere Arbeit jedoch berührt. Marco Krasselt und Christoph Baerwald aus Leipzig erläutern den gegenwärtigen Stand des Managements der Fibromyalgie, allgegenwärtig in unserem Alltag. Weg vom Häufigen zum Speziellen, aber auch Spannenden die Übersicht zum Thema Morbus Behçet durch Theodoros Xenitidis und Joerg Christoph Henes aus Tübingen. 
- Tab. 1 Entwicklungsstufen von Leitlinien (S1-S3) nach der Arbeitsgemeinschaft für medizinisch-wissenschaftliche Fachgesellschaften (AWMF) (www.awmf.de)

S1 Die Leitlinie wurde von einer Expertengruppe im informellen Konsens erarbeitet

S2k Eine formale Konsensfindung hat stattgefunden

S2e Sowohl eine systematische Evidenz-Recherche wie eine formale Konsensfindung hat stattgefunden

S3 Die Leitlinie hat alle Elemente einer systematischen Entwicklung durchlaufen

- Tab. 2 Evidenz- und Empfehlungsgrade nach dem Oxford Centre for Evidence-based Medicine (www.cebm.net)

\section{Evidenzgrade}

la Wenigstens eine Metaanalyse der Basis methodisch hochwertiger randomisierter, kontrollierter Studien (RCT)

Ib Wenigstens eine ausreichend große, methodisch hochwertige RCT

Ila Wenigstens eine hochwertige Studie ohne Randomisierung

Ilb Wenigstens eine hochwertige Studie eines anderen Typs (quasi-experimentelle Studien)

III Mehr als eine methodisch hochwertige nicht experimentelle Studie wie etwa Vergleichsstudien, Korrelationsstudien oder Fall-Kontroll-Studien

IV Meinungen und Überzeugungen von angesehenen Autoritäten, Expertenkommissionen, beschreibende Studien

\section{Empfehlungsgrade}

A „Soll“-Empfehlung auf Basis mindestens einer randomisierten kontrollierten Studie von insgesamt guter Qualität und Konsistenz ohne Extrapolation (Evidenzstufen la und Ib)

B „Sollte“-Empfehlung auf Basis einer gut durchgeführten, aber nicht randomisierten klinischen Studie (Evidenzstufen II oder III) oder Extrapolation von Evidenzebene I (bei fehlendem Bezug zur spezifischen Fragestellung)

C „Kann“-Empfehlung auf der Basis von Berichten von Expertenkreisen oder Expertenmeinungen und/oder klinischer Erfahrung anerkannter Autoritäten (Evidenzkategorie IV) oder Extrapolation von Evidenzebene Ila, Ilb oder III

Wir hoffen, dass wir Ihnen mit diesen Themen interessante Einblicke und neues Wissen zum täglichen Gebrauch bieten können.

Viel Spaß beim Lesen wünschen Ihnen

Ihre

Prof. Dr. med. Christoph Fiehn, Baden-Baden

Prof. Dr. med. Heinz-Jürgen Lakomek, Minden 